\title{
Optimal Routing based Load Balanced Congestion Control using MAODV in WANET Environment
}

\author{
Kanthimathi $\mathrm{S}^{1}$, Dr JhansiRani $\mathrm{P}^{2}$ \\ Department of Computer Science CMR Institute of Technology \\ VTU Research Center, Bengaluru, India
}

\begin{abstract}
A decentralized sort of network that can allow the nodes to communicate with them lacking any central controller is Wireless Ad hoc Networks (WANET). Network Congestions can befall on account of nodes' restricted Bandwidth (BW) together with dynamic topology. Network Congestions brings about data loss as it makes the Data Packets (DP) be dropped on the network. Therefore, in order to lessen Network Congestions, it is necessary to model Congestion Control (CC) systems aimed at the WANET. Thus, this paper offers an optimal routing centered CC scheme utilizing the Modified Ad hoc on-demand Distances Vector (MAODV) Routing Protocol (RP) aimed at the WANET. Here, primarily, the Source Node (SN) together with Destination Nodes (DN) is initialized, and after that, the MAODV discovers the multiple routing paths. Subsequently, Stochastic Gradients Descent Deep Learning Neural Network (SGD-DLNN) identifies the Congestion Status (CS) of every node in the discovered paths. In addition, the MAODV allocates the traffic over the optimum congestion-free routing path if congestion befalls. The Levy Flight Based Black Widow Optimization (LF-BWO) algorithm chooses the optimal routing paths as of congestion-free paths. Centered upon path lifetime, residual energy, link cost, together with path distance, this algorithm enhances the Data Transmission (DT) performance by means of discovering a path. The experimentation's outcomes are rendered to exhibit the proposed RP's effectiveness.
\end{abstract}

Keywords-Routing; congestion control; Wireless Ad Hoc Networks (WANET); Modified Ad hoc on-demand Distance Vector (MAODV); Levy Flight based Black Widow Optimization (LFBWO); Stochastic Gradient Descent Deep Learning Neural Network (SGD-DLNN)

\section{INTRODUCTION}

WANETs consist of various mobile wireless nodes that could travel arbitrarily with the capacity to connect or depart the network [1]. A WANET can be utilized in various applications, namely, disaster recovery, search, and also rescue operations. The specific characteristics of WANET are asymmetry, dynamic network topology, multiple-hop communication, along with limited BW as well as energy resources [2]. Obstruction is occurred in WANETs on account of the Packet Loss (PL), and it can well be effectively reduced by involving a $\mathrm{CC}$ scheme, which includes a routing algorithm and flow control on a network layer [3]. The intensive streaming traffic in WANETs can result in more packet loss, longer delay, and Quality of Service (QoS)-related performance degradation caused by congestion. When the present traffic load surpasses the existing transmission ability at every point within the network, congestion takes place. Congestion has a completely adverse ramification on the
WANET's performance $[4,5]$. Congestion particularly obtains the excess of node buffers, the degradation of the overall channel quality, and the increase of both loss rates and transmission delays [6].

Different methods are created, which tells the SN about the CS for augmenting the capacity of WANETs and to lessen clogging. An SN retransmits or delays the transmission in accordance with the CS. Packets are circulated evenly amongst every node which participates in transmission [7]. A series of specific congestion-related issues had been detected and also located, involving intense Throughput (TP) degradation as well as immense fairness problems [8]. The end-to-end $\mathrm{CC}$ has a strong reliance on round-trip time, which certainly leads to PL. In contrast, the hop-by-hop CC protocol has a faster response speed [9]. Therefore, the best way is chosen to avoid the $\mathrm{CC}$. Routing is the process of choosing a suitable path from SN to DN to send DP [10]. Analysis of traditional mobile ad hoc RP shows that these protocols are not efficient for Wireless networks [11].

The utmost significant reasons of these topology-centered RP explicitly, Dynamic Sources Routing (DSR), Ad hoc on Demands Distances Vectors Routing (AODV) [12], along with Optimized Links-States Routing (OLSR) [13] is that their route uncertainty owing to the higher speed vehicle nodes [14]. Ad hoc On-Demand Multi-path Distances Vector (AOMDV) is amongst the most well-known WANET reactive-RP [15, 16, and 17]. Hence, researchers have extensively modified this protocol to enhance their performance [18]. The multipath concept is utilized by the AOMDV in the routing process. SN chooses the shortest way to a DN. The selection of congested nodes inside an active path degrades network performance which lessens Packet Delivery Ratios (PDR), TP, PL and increases overhead in the network. Congested nodes take up more power resources of the network. Therefore, to develop the performance parameters within the network, an effectual CC is needed [19, 20]. An effective Congestion Detection (CD) and optimal routing-based $\mathrm{CC}$ is proposed by this paper in a WANET environment.

\section{RELATED WORK}

Devarajan Krishnamoorthy et al. [21] suggested an efficient CC system for MANET. An algorithm was utilized by the system to resolve the congestion problem centered on the Relative Traffic Links Matrix Routing to obtain a solution with an enhanced PDR along with decreased overhead. The selected traffic matrix technique's effectiveness was examined 
by contrasting its performance with Capacity Optimized Cooperative communications (COCO). The traffic matrix method considerably enhanced the accuracy to acquire the traffic patterns on MANET as suggested by the experiments. The simulation outcomes had shown that the system performed well when contrasted to the COCO method. But the system offered a low efficiency and had a certainty environment.

R. Vadivel and V. Murali Bhaskaran [22] offered an adjustable dependable and $\mathrm{CC}$ protocol for MANET. The shortest route was found for effective DT among the many paths which were constructed. The congestion was identified through the employment and capability of links and paths. When congestion was detected by $\mathrm{SN}$ on a link along the route, it had spread traffic over the different routes by regarding the path availability threshold and used a traffic splitting function. If the congestion was not solved by a node, it signaled its neighbors based on the congestion's indication bit. The system showed that it was dependable and achieved more TPs with abridged packet drops and also overhead as per the simulation. But the system was somewhat difficult to prove the system's performance when contrasted with less existing methodologies.

Jogendra Kumar et al. [23] introduced a CC load balancing adjustable RP for a random waypoint model in MANET to decrease delay, system routing overhead, congestion, and improved the life of network in MANET. Every mobile node in the system considered the newest traffic load and preserved an estimated record for every locality in the transmitting table called the locality table. The system was contrasted with the current RP of MANET with respect to TP, end-to-end delay (EED), packet drops average jitter, PDR, and normalized routing overhead on the network. This system exceeded the existing methods, but the system was unable to identify the congestion.

M. S. Gowtham et al. [24] presented a CC and also packet recovery aimed at the cross-layer method in MANET. The characteristics of this model were localized packet recovery, deterministic, ability to trade-off efficiency, and peer-to-peer recovery. The system was able to reclaim the lost packets via storing a duplicate of the packet. Data traffic congestion rates of high as well as low priority packets were maintained by allocating priority orders to the packets. The higher priority flow would be developed to get desired access to the medium and its flow rate of low priority was altered to have a high flow rate of higher priority. Therefore, the total performance had provided a better outcome than the prevailing method.

Ammar Alhosainy et al. [25] recommended a joint optimal congestion, multiple-path routing, along with contentions control for WANET. A splitting factor was utilized by the system to replace the linearity in the relationship within each session and its multipath routes that extended earlier models to include routing over potentially multiple paths in the optimization framework. The new variable, proper transformation and the used Ohm's law analogy led to a convex and decoupled optimization framework that found the optimum solution in a distributed form. A distributed algorithm that found the best solution for general concave utility functions was utilized by the system. The results have exhibited that the system had achieved an optimum solution when contrasted with conventional methods. But the costs of delay, queue length, along with available energy in each node were not taken into consideration, thus better route decisions were not given by the system.

Amit Sharma and Khushboo Pawar [26] presented an approach called CRAODV which was utilized for $\mathrm{CC}$ in mobile ad-hoc networks. The Setup process was started at each node as an agent. A process running on the node was the agent and started with the routing agent. $\mathrm{CD}$ was the mechanism utilized for recognizing the problem that had already occurred or else going to occur. The final action taken was the Congestion Handling / Optimal route discovery Mechanism, with which the node or the sink gave feedback to the network to take some action relative to the problem and Congestion aware scheme. This scheme was affected by hopto-hop CD, in contrast, to end to end. The system had provided improved performance with reference to packet drop ratio as per the simulation results. But the system had focused on only ' 1 ' metric. It was not contrasted with other qualitative metrics.

\section{PROposed Optimal Routing BASED CONGESTION CONTROL IN WANET}

A WANET is basically a wireless network that functions free of any fixed infrastructure. Here, the nodes function both as the hosts and the routers forwarding DP. Ad hoc networks (AHN) are primarily employed in military applications, disaster recoveries, together with emergency operations owing to their self-organizing manner. The Network Congestions occurs on account of the nodes' restricted BW together with dynamic topology. If a network node or link is carrying more data than it can handle, there is a chance for Network Congestions, which will in-turn affect the communication by initiating data loss. An apt way must be adopted to model a CC system that can evade the PL on the WANET. Thus, this paper proposes a $\mathrm{CD}$ and optimal routing-centered $\mathrm{CC}$ scheme aimed at WANET. The proposed work encompasses ' 5 ' phases: i) node initialization, ii) $\mathrm{SN}$ and $\mathrm{DN}$ generation, iii) route discovery, iv) CS identification, and v) load-balanced $\mathrm{CC}$ centered on optimal routing path selection. These phases are elucidated below in a meticulous manner. Fig. 1 evinces the flow of the proposed Congestion control system.

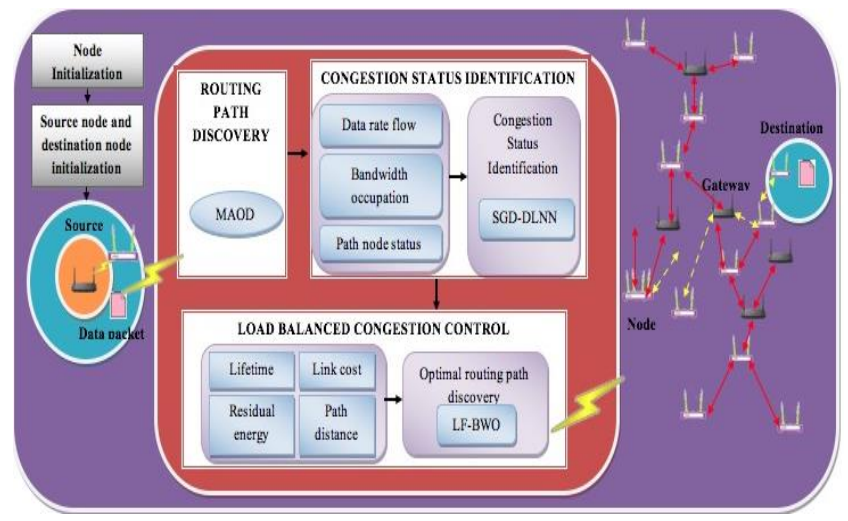

Fig. 1. Block Diagram of Proposed Congestion Control Algorithm. 


\section{A. Node Initialisation}

At first, the wireless nodes along with their counts are initialized. A wireless node is basically an internet-linked device placed in the WANET, and its location along with the point of attachment to the internet might regularly be varied. The wireless node $\left(N_{k}\right)$ is exhibited as,

$\mathrm{N}_{\mathrm{k}}=\left\{\mathrm{N}_{1}, \mathrm{~N}_{2}, \mathrm{~N}_{3}, \ldots \ldots, \mathrm{N}_{\mathrm{N}}\right\}$

Wherein, $\mathrm{N}_{\mathrm{N}}$ implies the n-number of wireless nodes.

\section{B. Initialisation of Source and Destination Node}

The SN along with DN is defined here. The network is self-configuring as the nodes could connect or leave it and move without restraint according to their need. If a node $N_{s}$ transmits a DP to a node $N_{d}$, the $\mathrm{SN}$ and DN are exhibited as,

$\left(\mathrm{N}_{\mathrm{s}}, \mathrm{N}_{\mathrm{d}}\right) \in \mathrm{N}_{\mathrm{k}}$

Wherein, $\mathrm{N}_{\mathrm{s}}$ and $\mathrm{N}_{\mathrm{d}}$ signifies the source and destination nodes.

\section{Routing Path Discovery}

Here, a routing path for sending a DP as of the SN to DN is discovered. Most $\mathrm{ADN}$ mainly focuses on discovering a single routing path betwixt the $\mathrm{SN}$ and $\mathrm{DN}$, which is not efficient. If the primary path breaks or NC occurs, then the intermediate nodes will immediately drop the packets since there are no alternating paths available for the destination. Thus, the proposed system finds manifold routing paths betwixt SN and DN. The proposed work employs the MAODV protocol for discovering all the probable paths to the DN. In the MAODV, the routing path discovery commences with the generation of the Route Request (R_REQ) packet. R_REQ packet is sent to the neighboring node once an SN wishes a path to the DN and then waits on the Route Reply (R_REP) packet as of the DN. The R_REQ packet format is exhibited in Table I.

TABLE I. PACKET FORMAT OF R_REQ

\begin{tabular}{|l|l|l|l|l|l|}
\hline $\begin{array}{l}\text { Source } \\
\text { Address }\end{array}$ & $\begin{array}{l}\text { Source } \\
\text { Sequence } \\
\text { Number }\end{array}$ & $\begin{array}{l}\text { Broadcast__ } \\
\text { id }\end{array}$ & $\begin{array}{l}\text { Destination } \\
\text { Address }\end{array}$ & $\begin{array}{l}\text { Destination } \\
\text { Sequences } \\
\text { Number }\end{array}$ & $\begin{array}{l}\text { Hop_ } \\
\text { Count }\end{array}$ \\
\hline
\end{tabular}

Once the neighbor node attains the R_REQ packet, it accepts the request for instantaneous transmission. Then, a reverse path to the $\mathrm{SN}$ is set by means of taking the former hop of the R_REQ as the subsequent hop. In a similar manner to the intermediate nodes, a reverse-path is set by the $\mathrm{DN}$ subsequent to attaining the initial copy of a R_REQ packet. As the R_REP advances towards the SN, it institutes a forward path to the DN at every hop. Table II evinces the packet format of R_REP.

TABLE II. PACKET FORMAT OF R_REP

\begin{tabular}{|l|l|l|l|l|}
\hline $\begin{array}{l}\text { Source } \\
\text { Address }\end{array}$ & $\begin{array}{l}\text { Destination } \\
\text { Address }\end{array}$ & $\begin{array}{l}\text { Destination } \\
\text { Sequence } \\
\text { Number }\end{array}$ & Hop_Count & Life_Time \\
\hline
\end{tabular}

An SN might get manifold R_REP messages with disparate paths. The routing entries will be updated presuming that the R_REP has a bigger sequence number, that is, fresh information. In MAODV, once the path is chosen, it will not be expired until the DT is finished. Thus, all the probable routing paths betwixt $\mathrm{SN}$ and $\mathrm{DN}$ are discovered.

In general, the AODV for the intention of storing routing information employs Routing Tables (RT). These encompass the subsequent fields (columns): source address, source sequence number, broadcast id, a destination address, destination sequence number, hop_count, and life_time. However, in the proposed work, ' 3 ' more columns: $D R$, Path Node (PN) status, together with BW Size are included. Here, the Mobile Agent (MA) is initialized to monitor and update the $D R$, PN status, and BW size of all nodes on the AODV aimed at CS identification. Thus, the MA is joined with the AODV. This protocol is labelled as MADOV. The RT of MAODV encompasses the subsequent fields:

<source_address,source_sequence_number,broadcast_id,desti nation_address,destination_sequence_number,hop_count,life_ time,data_rate,bandwidth_size, path_node_status>

In the MAODV, the RT is maintained on every node. The discovered multiple routing paths by means of MAODV is mathematically represented as,

$\mathrm{P}(\mathrm{R})_{\mathrm{k}}=\left\{\mathrm{P}(\mathrm{R})_{1}, \mathrm{P}(\mathrm{R})_{2}, \ldots \ldots \ldots \mathrm{P}(\mathrm{R})_{\mathrm{n}}\right\}$

Wherein, $P(R)_{k}$ implies the discovered routing paths and $P(R)_{n}$ signifies the n-number of routing paths. The SN, by utilizing these routing paths, sends DP to DN. NC can occur at any time, which leads to PL when the number of DP reaching the node surpasses its buffer capacity. It is vital to make out the CS of every node on the routing paths beforehand to evade such congestion, which is estimated in the subsequent phase.

\section{Congestion Status Identification}

To find the whole traffic present across disparate routing paths, the CS of every node should be calculated which is done by this phase. The total packets in the queue at any specified time $t$ is the Queue Load (QL) of a node. NC will befall when the QL augments. Thus, the QL ought to be kept to a least so as to evade this. It is vital to know the nodes' CS in the network for keeping the QL minimum. The proposed work utilizes ' 3 ' metrics: $D R$, PN status, and $\mathrm{BW}$ size for identifying the CS of all nodes.

1) Path node status: The MA monitors the PN-status on the base of its communication range. Here, to detect if the node is dropping or forwarding the packets, the PN-status is monitored as well as estimated.

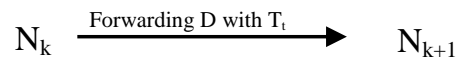


Wherein, $D$ signifies the data packet and $T_{t}$ implies the DT time. The PN-status is identified centered on the subsequent conditions.

if $\mathrm{N}_{\mathrm{k}}$ forward $\mathrm{D}$ to $\mathrm{N}_{\mathrm{k}+1} \& \& \mathrm{~T}_{\mathrm{d}}>=\mathrm{T}$

Path_Node_Trust $=$ Low

if $\mathrm{N}_{\mathrm{k}}$ drops $\mathrm{D}$ or $\mathrm{T}_{\mathrm{d}}>\mathrm{T}$

Path_Node_Trust $=$ High

Wherein, $\mathrm{T}$ signifies threshold value. The proposed method has a fixed ' 1 ' threshold value, when $T_{d}$ of the node is larger than that threshold value or $\mathrm{N}_{\mathrm{k}}$ failed to forward the packet to $\mathrm{N}_{\mathrm{k}+1}$, the PN trust level is high, otherwise low.

2) Data rate: The total DP transmitted amid a particular time period $t$ over a network is termed the DR. To evade congestion, there should be a balance betwixt the SN and DN by means of knowing the QL at SN and DN. The DR of the knumber of nodes is computed by,

$\mathrm{DR}_{\mathrm{k}}=\mathrm{A}(\mathrm{D}) / \mathrm{t}$

Wherein, A(D) signifies the amount of DP. DR should be on the lower side for lossless packet transmission. The SN, by means of augmenting the DR, should quickly send the packets once the DP-load augments. Thus, if the DR is augmented, then the DP-load at that node is higher, which can bring about NC. Centered on a fixed threshold value, the DR status of every node is computed similar to the QL.

if $\mathrm{DR}_{\mathrm{k}}>=\mathrm{T}$

Data_rate_flow $=$ High

if $\mathrm{DR}_{\mathrm{k}}<\mathrm{T}$

Data_rate_flow $=$ Low

3) Bandwidth rate: The potential of a particular node to send as well as receive data in a precise period of time is called the Node BW rate. Amid the DT, the QL augments once congestion happens, and when it goes beyond the threshold, the BW occupation of the specific node will be augmented. Thus, the node must have the least BW occupation for a congestion-free node. The BW of the knumber of nodes $\left(B W_{k}\right)$ is expressed mathematically as,

$\mathrm{BW}_{\mathrm{k}}=\left\{\mathrm{BW}_{1}, \mathrm{BW}_{2}, \mathrm{BW}_{3}, \ldots \ldots \mathrm{BW}_{\mathrm{N}}\right\}$

Wherein, $B W_{N}$ implies the 'N'-number of $\mathrm{BW}$ rate. The BW rate status is computed utilizing the subsequent conditions.

if $\mathrm{BW}_{\mathrm{k}}>=\mathrm{T}$

Bandwidth_rate_occupation $=$ High

if $\mathrm{BW}_{\mathrm{k}}<\mathrm{T}$

Bandwidth_rate_occupation $=$ Low

Aimed at diverse factors like PN status, DR, and BW rate, the fixed threshold value is changed. Centered on these ' 3 ' factors, each node's CS is identified. Every time the node receives a R_REQ packet as of the other node, their DR and $\mathrm{BW}$ rate are supervised by the MA in the MAODV that updates this information into the RT. After enumerating every node's DR and BW rate, the final outcome, explicitly, the node's CS is predicted by using the Deep Learning Neural Network (DLNN).

4) Stochastic gradient descent deep learning neural network: DLNN originated from the Artificial Neural Network (ANN) family and it is considered to comprise more than one Hidden Layer (HL). Incrementing the number of HLs can lessen the neural network training error. The various HLs (deep learning) train themselves to process as well as study the data intensely by the filtering of information via the multiple HLs. If the neural network studies intensely, then the detection performance will automatically increment. It is the feedforward network that is normally trained by employing the back-propagation technique. However, this back-propagation design causes higher training time. The Stochastic Gradient Descent (SGD) weight updating can be hybridized with the DLNN for evading the back-propagation issues by executing weight updating process in DLNN. Hence, the proposed scheme is called "SGD-DLNN". SGD-DLNN's common structure is showcased in Fig. 2. The proposed SGD-DLNN is trained to find the nodes' CS centered on various rules that are given in the Table III as:

For CS identification, at first, the $D R$, BW rate, along with the PN status values are inputted into the input layer neurons. After getting the inputted values, corresponding weight values are arbitrarily created aimed at every input. The inputted values as well as their equivalent weight values are articulated as,

$$
\begin{aligned}
& C_{k}(F)=\left\{C_{1}(F), C_{2}(F), C_{3}(F), \ldots \ldots ., C_{N}(F)\right\} \\
& C_{k}(F) \rightarrow \sum\left(D R_{k}, B W_{k}, N S_{k}\right) \\
& \psi_{k}=\left\{\psi_{1}, \psi_{2}, \psi_{3}, \ldots \ldots ., \psi_{N}\right\}
\end{aligned}
$$

Here, $\mathrm{C}_{\mathrm{K}}(\mathrm{F})$ signifies the $\mathrm{CD}$ factors like $\mathrm{DR}, \mathrm{BW}$ rate, along with the PN status, and $\psi_{k}$ implies the arbitrarily initialized weight values. For evading back-propagation issue, the weight values have been initialized utilizing the SGD as,

$\hat{\psi}_{k}=\psi_{k}+\chi$

Here, $\hat{\psi}_{k}$ signifies the novel weight values initialized via SGD and $\chi$ stands for the step size that is computed as,

$$
\chi=G_{d} * \gamma
$$

Here, $\mathbb{G}_{d}$ implies the inputted value's gradients and $\gamma$ signifies the learning rate. Low learning rates make the algorithm to reach nearer to the targeted output. Thus, always it is better to glue to a lesser learning rate like 0.01 . 
TABLE III. RULES INVOLVED IN CONGESTION STATUS PREDICTION

\begin{tabular}{|l|l|l|l|}
\hline \multicolumn{2}{|l|}{ Rule Details } & \multirow{2}{*}{ Congestion Status } \\
\cline { 1 - 1 } $\begin{array}{l}\text { Data Rate } \\
\text { Flow }\end{array}$ & $\begin{array}{l}\text { Bandwidth } \\
\text { Occupation }\end{array}$ & $\begin{array}{l}\text { Path Node } \\
\text { Trust }\end{array}$ & \\
\hline High & High & High & High Congestion \\
\hline High & High & Low & High Congestion \\
\hline High & Low & High & Medium congestion \\
\hline High & Low & Low & High Congestion \\
\hline Low & Low & Low & Medium Congestion \\
\hline Low & Low & High & No Congestion \\
\hline Low & High & Low & Medium Congestion \\
\hline Low & High & High & Medium Congestion \\
\hline
\end{tabular}

After attaining the updated weight values, the inputted values $\mathrm{C}_{K}(\mathrm{~F})$ and the novel weight values $\hat{\psi}_{k}$ have been provided to the HL. Next, these ' 2 ' values are individually multiplied and after that those values are completely added in the HL.

$\Phi(h)_{k}^{i}=\sum_{k=1}^{N} C_{k}(F) \hat{\psi}_{k}$

Here $\Phi(h)_{k}^{i}$ symbolizes the input of the HL. Next, the Gaussian activation function is created in the hidden nodes, which is articulated as,

$G\left(\Phi(h)_{k}^{i}\right)=\exp \left(\Phi(h)_{k}^{i^{2}}\right)$

Here, $G(\bullet)$ signifies the Gaussian activation function. The HL's output is computed centered on the $G(\bullet)$ together with the bias value $\phi$.

$\Phi(h)_{k}^{o}=\phi+\sum G\left(\Phi(h)_{k}^{i}\right)$

Here, $\Phi(h)_{k}^{o}$ implies the HL's output. At last, in the outputted layer, the total inputted signals' weights are summed aimed at obtaining the value of outputted layer neurons.

$\Phi(o)_{k}^{o}=\phi+\sum \Phi(h)_{k}^{o} \hat{\psi}_{k}$

Here, $\Phi(o)_{k}^{o}$ signifies the classifier output unit that comprises ' 3 ' classes: High congestion, medium congestion, and low congestion. Centered on the rules mentioned above, each node's CS is identified. After that, the load-balanced CC is done to evade PL.

\section{E. Load Balanced Congestion Control}

The routing protocol's potential to balance the traffic amid the multiple routing paths is called Load balancing. If the SN desires to converse with the $\mathrm{DN}$, it examines its $\mathrm{RT}$ aimed at a valid routing path to the DN. If it is identified, the MAODV examines the congestion status of the PN utilizing the SGDDLNN. If congestion is present in any node, the paths that comprise those congested nodes are evaded aimed at the upcoming packet transmission until the attainment of standard traffic condition. The MADOV begins to distribute the traffic over the other congestion-free paths for attaining the normal traffic condition aimed at evading congestion. For achieving a lossless packet transmission, minimal transmission delay, minimal energy consumption, along with the maximal $\mathrm{TP}$, the routing paths must be highly energy-efficient, consistent and shortest as of the SN to the DN. Hence, for obtaining an optimal routing path, the proposed MADOV employs LFBWO Algorithm.

An optimal routing path discovery is employed aimed at spotting the effective link quality path for transferring data as of the SN towards the DN. The LF-BWO technique is implemented aiming at the optimal routing path discovery. The proposed LF-BWO chooses the optimal path centered on the factors namely lifetime, link cost, residual energy, and route distance, which are elucidated as,

1) Life time: The lifetime of routing path is alternatively stated as to which extent the path survives in the entire network that is enumerated as,

$$
L_{f}=\rho\left(L\left(t_{0}\right)+L\left(t_{0}+t_{\max }\right)\right) ; \quad t_{\max }=t_{p 1 p 3}
$$

Here, $\rho\left(L\left(t_{0}\right)+L\left(t_{0}, t_{\max }\right)\right)$ signifies the probability that the path continuously will be available as of time $t_{0}$ to $\left(t_{0}+t_{\max }\right), L\left(t_{0}\right)$ and $L\left(t_{0}, t_{\max }\right)$ represents the path availability whilst ' 2 ' nodes' velocity remained unaltered and altered, correspondingly and $t_{p 1 p 3}$ signifies the time consumed by the node $N_{1}$ for travelling as of node position $p 1$ to $p 3$.

$$
\begin{aligned}
& L\left(t_{0}\right)=\exp \left(-2 \eta t_{\text {max }}\right) \\
& L\left(t_{0}+t_{\text {max }}\right)=\frac{1-\exp \left(-2 \eta t_{\text {max }}\right)}{2 \eta t_{\text {max }}}+\frac{\eta t_{\text {max }} \exp \left(-2 \eta t_{\text {max }}\right)}{2}
\end{aligned}
$$

Here, $\eta$ stands for the carrier's wavelength.

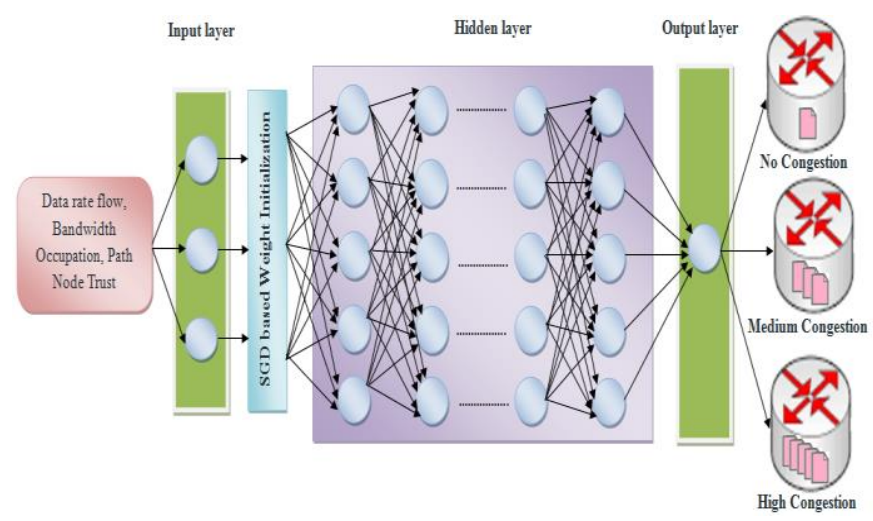

Fig. 2. Structure of SGD-DLNN.

2) Link cost: The Link Cost has been measured by splitting the reference $\mathrm{BW}$ via the interface $\mathrm{BW}$. The full cost 
towards the $\mathrm{DN}$ is the individual links' cost in the path towards destination.

$$
L_{c}=\sum_{k=N_{s}}^{N_{d}}\left[\frac{B W_{R}}{B W_{I}}\right]_{k}
$$

Here, $B W_{R}$ and $B W_{I}$ represents the reference $\mathrm{BW}$ and interface BW.

3) Residual energy: The energy which stayed past the packet transmission is termed the Residual Energy, which is,

$E_{R}=E_{I}-E_{C}$

Here, $E_{R}$ signifies the Residual Energy, $E_{I}$ stands for initial energy and the $E_{C}$ signifies the consumed energy throughout the DT. The $E_{C}$ is articulated as,

$E_{C}=N_{D} \times\left(E_{T}+E_{P}\right)$

Here, $N_{D}$ implies the number of DP and the $E_{P}$ represents the energy needed aimed at packet processing and the $E_{T}$ symbolizes the transmitting energy aimed at a packet that is articulated as,

$E_{T}=\frac{D_{s} \times D_{T P}}{B W}$

Here, $D_{s}$ indicates the DP size, $D_{T P}$ signifies the packet transmitting power, and the $B W$ signifies the wireless link BW.

4) Path distance: The distance betwixt the $\mathrm{SN}$ and the $\mathrm{DN}$ is the route distance, which is articulated as,

$R_{d i s t}=N_{H}\left(N_{s}, N_{d}\right)$

Here, $R_{\text {dist }}$ signifies the route distance and the $N_{H}\left(N_{s}, N_{d}\right)$ represents the number of hops betwixt node $N_{s}$ and $N_{d}$, respectively. The lowest hop count signifies the smallest routing path. The least distance paths offer rapid DT along with less processing time.

The ' 4 ' factors mentioned above are fixed as the fitness function aimed at the LF-BWO method for locating the optimal routing path. BWO has been enthused by the black widow spiders' distinct mating features. The BWO sustains a balance betwixt the exploitation as well as exploration phases, and also offers fast convergence speed; as well as evades the local optima issue. Nevertheless, a fast convergence speed can't be assured aimed at every databases type owing to the random parent selection. To avoid this issue, the Levy Flight (LF) distribution is utilized in the BWO algorithm for parent selection. So, the proposed protocol is termed LF-BWO.
At first, the black widow population is structure as an array that is articulated as,

$$
A\left[Y_{\text {widow }}\right]=\left[Y_{1}, Y_{2}, Y_{3}, \ldots Y_{N}\right]
$$

Here, $A\left[Y_{\text {widow }}\right]$ signifies the population array and is regarded as $p_{p 1}$. After that compute each black widow's fitness; in which, all black widow solution is regarded as paths. Hence, each solution's fitness is regarded as the maximum lifetime, maximum residual energy, minimum link cost, and minimum path distance, that is articulated in Eq. (31),

$F_{\text {opmial path }}=\left\{\begin{array}{ll}\max \left(L_{f}\right) ; & \text { Maximum life time } \\ \max \left(E_{R}\right) ; & \text { Maximum residual energy } \\ \min \left(L_{c}\right) ; & \text { Minimum link cost } \\ \min \left(R_{\text {dist }}\right) ; & \text { Mininum route distance }\end{array}\right\}$

After evaluating the fitness, all candidates are sorted by their fitness value and then stocked in $p_{p 1}$. Then parents' pairs are chosen utilizing the LF Distribution aimed at executing the procreating process. In LF-BWO's every generation, the most excellent individuals are chosen as parents $Y_{1}$ and $Y_{2}$ in accordance to LF Distribution.

$$
L(Y)=t(-Y), \quad 1<Y<3
$$

Here, L(Y) signifies the LF Distribution. Next children pair is generated by employing the below equation,

$$
\begin{aligned}
& o f f_{1}=\varpi \times Y_{1}+(1-\varpi) \times Y_{2} \\
& o f f_{2}=\varpi \times Y_{2}+(1-\varpi) \times Y_{1}
\end{aligned}
$$

Here, off 1 and $o f f_{2}$ represents the children pair; this procedure has been repeated aimed at $N / 2$ times. After that, the children along with the mom are sorted via their fitness value and then added on to the array $\left(p_{p_{2}}\right)$. In accordance with which the number of survivors is set on, fix the cannibalism rating (CR). The fitness value is utilized for deciding the weak and strong black widows. Demolish the weaker solutions and then restore the remaining solution in the $p_{p_{2}}$. After the selection of one solution as of the $p_{p 1}$, mutate that solution's one chromosome and then create a novel solution; save the new solution in the $p_{p 3}$. At last, $p_{p_{2}}$ and $p_{p 3}$ solutions are stocked in the novel array $O_{p_{p}}$.

$O_{p_{p}}=p_{p_{2}}+p_{p_{3}}$

As of the $O_{p_{p}}$ array, the finest solution is chosen by analogizing each solution's fitness values. The LF-BWO method's Pseudocode is displayed below in Fig. 3. 
Input: Selected Routing Paths

Output: Optimal Routing Path

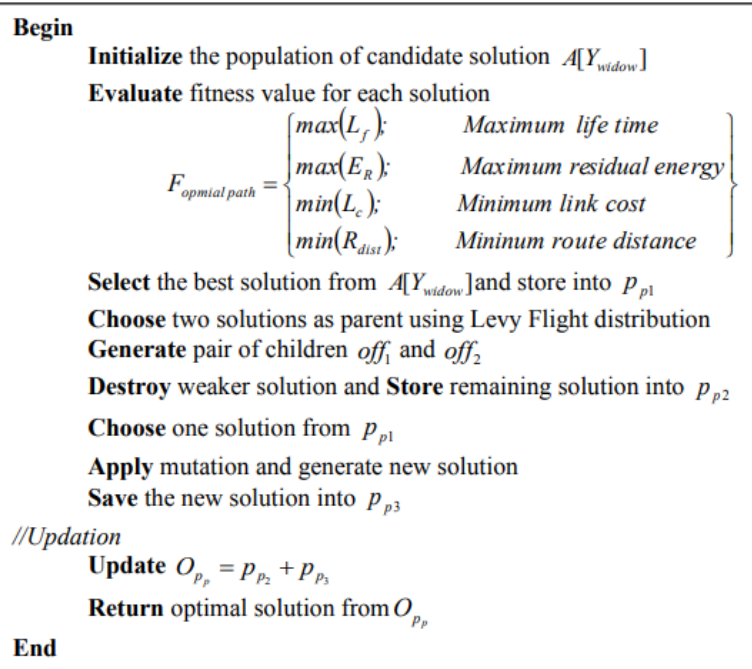

Fig. 3. Pseudocode of LF-BWO.

Thus, every path's fitness value can be analyzed and analogized with the fitness values of another path; and then the optimal routing paths are chosen centered upon the fitness values. The optimal path means the path which comprises a higher lifetime, higher energy level, low link cost, and a lesser number of hops. Priority can be offered to the energy level and lifespan. If the routing path comprises the greatest lifespan along with energy level however doesn't comprise the least link cost and distance, it is chosen nevertheless with low priority. At last, the routing process commences to spread the traffic packets over the chosen optimal paths. The packet drop is drastically evaded by transmitting DPs via the optimal congestion-free routing paths. Fig. 4 exhibits the routing path's optimal selection.

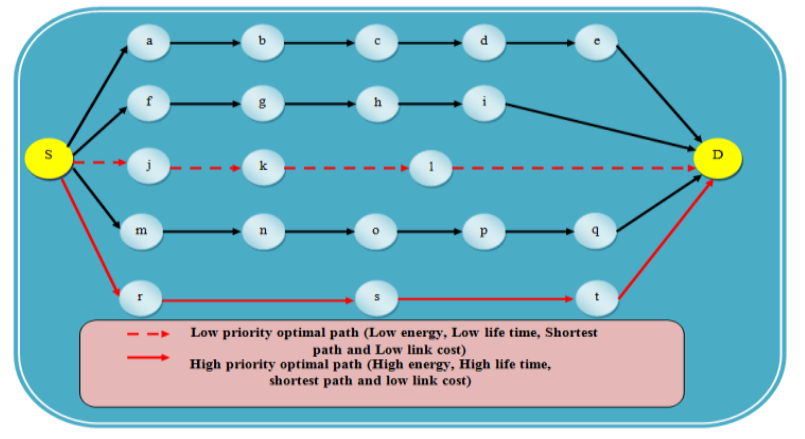

Fig. 4. Optimal Routing Path Selection.

\section{RESULTS AND DISCUSSIONS}

Here, the proposed Congestion Control system's performance utilized aimed at PL avoidance between the SN and the DN is analyzed by performing the different experiments on the Network Simulation Tool-Version 2 (NS2). The proposed MADOV performance is weighted against few conventional existent RPs centered on a few quality metrics.

\section{A. Performance Metrics}

The MADOV's performance is analyzed centered on the below metrics,

1) Packet Delivery Ratio: It is the ratio betwixt the number of DPs received by the destination and the whole number of DP sent as of the source.

2) End to End Delay: It is regarded as the time that is utilized aimed at the transmission of a DP as of the SN to DN over the WANET.

3) Energy Consumption: Energy consumption elucidates the amount of energy consumed by the network nodes aimed at transmitting a DP as of the source towards the destination.

4) Throughput: TP defines the average efficient delivery of DPs to the destination in specific simulation time.

5) Reliability: Reliability determines the metric that calculates the system's lifespan aimed at a period. It is reciprocally proportional to the PL.

Here, the PDR, EED, energy consumption, TP, and reliability of the proposed and existent RPs are calculated and the comparative examination of proposed and existent RPs is provided below.

\section{B. Comparative Performance Analysis}

The experimental setup for the network is mentioned in Table IV.

The proposed MADOV's performance is weighted against the conventional RPs like the AODV-RP, DSR, Temporally Ordered Routing Algorithm (TORA), and the BW-Aware Routing Strategy (BARS) in reference to PDR, EED, energy consumption, TP, and reliability.

Fig. 5 exhibits the performance analogy of the proposed and existent RPs with regard to PDR. Whilst the PDR is high, the DP transmission's performance attains more proficiency, and the transmitted DPs are sent to the destination with no PL. The PDR is computed by changing the time as of $10 \mathrm{~ms}$ to 50ms. In which, the MAODV attains a PDR of 95\%, 95.5\%, $96.2 \%, 96.7 \%$, and $96.9 \%$. However, the existent RPs attained a low PDR analogized with the MAODV. Even, no existent RP attained a PDR of more than $93 \%$. This outcome clarified that the MAODV sent the DP to the destination successfully analogized with the other RPs.

Fig. 6 exhibits the examination of the EED of the MAODV and the existent AODV, DSR, TORA, and BWRS. In the BWRS, the delay is extremely high; whereas the AODV, DSR, and TORA comprise a minimal delay than the BWRS. Nevertheless, the proposed MAODV's EED is much less than the AODV, DSR and, TORA. Aimed at various times, the EED can be incremented although the proposed MAODV's delay is less analogized with the existent RPs as the MAODV suggested the optimal routing paths aimed at communication. By utilizing the optimal paths, the transmission delay is drastically decremented. Hence, the MAODV is proficient of delivering enhanced performance over the AODV, DSR, TORA, as well as BWRS on EED. 


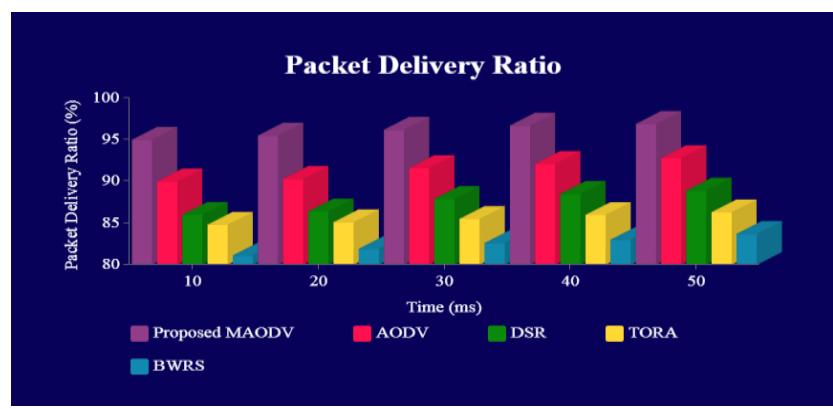

Fig. 5. Comparison of Packet Delivery Ratio for different Protocols.

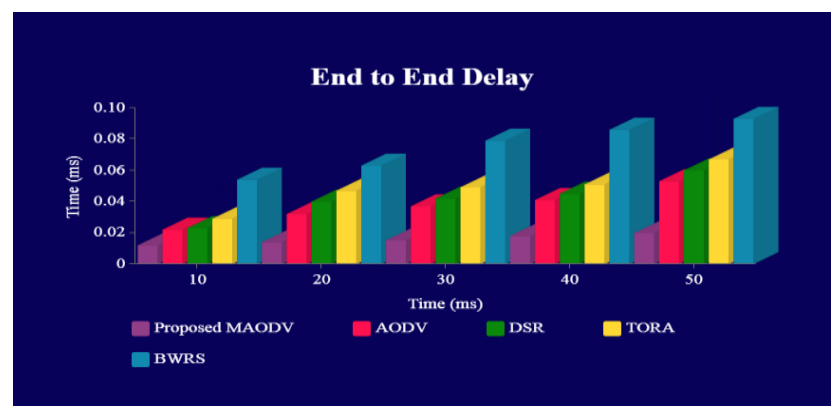

Fig. 6. Comparison of End to End Delay for different Protocols.

TABLE IV. EXPERIMENTAL SETUP

\begin{tabular}{|l|l|l|}
\hline S. No & Parameters & Values \\
\hline 1 & Simulator & NS 2 \\
\hline 2 & Network area & $1500 \mathrm{~m} \times 1500 \mathrm{~m}$ \\
\hline 3 & Propagation & Two Ray Ground \\
\hline 4 & Packet Size & 500 bytes \\
\hline 5 & Routing Protocol & MAODV \\
\hline 6 & Speed of node & $0-20 \mathrm{~ms}^{-1}$ \\
\hline 7 & Simulation time & $100 \mathrm{~s}$ \\
\hline 8 & Mobility Speed & $2-20 \mathrm{~m} / \mathrm{s}$ \\
\hline
\end{tabular}

Fig. 7 explicates the graphical performance examination of the proposed and existent RP in reference to energy consumption. The network's energy consumption is calculated in terms of Jules (J). All the RPs' energy consumption is enumerated centered on the variation of the number of nodes as of 20 to 100 . The proposed MAODV's energy consumption for 20 nodes is $1.2 \mathrm{~J}$, but the existent AODV, DSR, TORA, and BWRS comprise an energy consumption of $1.6 \mathrm{~J}, 1.9 \mathrm{~J}, 2.1 \mathrm{~J}$, and $2.29 \mathrm{~J}$ that is more than the proposed RP. For efficient DT, the network's energy consumption must be less for preventing the nodes as of a network failure. Aimed at the remaining number of nodes also, the MAODV consumes lesser energy analogized with the other RPs.

Fig. 8 exhibits the performance analogy of the MAODV and existent RPs concerning reliability. Reliability is enumerated with regards to percentage. Aimed at efficient DT's performance, reliability must be high. Here, the reliability performance is taken aimed at the various time periods, and then the reliability is linearly augmented. Whilst reaching $50 \mathrm{~ms}$, the MADOV's reliability is $98 \%$, but the AODV, DSR, TORA, and BWRS attain the reliability of $94 \%$, $85 \%, 75 \%$, and $73 \%$, correspondingly. These outcomes exposed that the network is extremely effective whilst employing the MAODV.

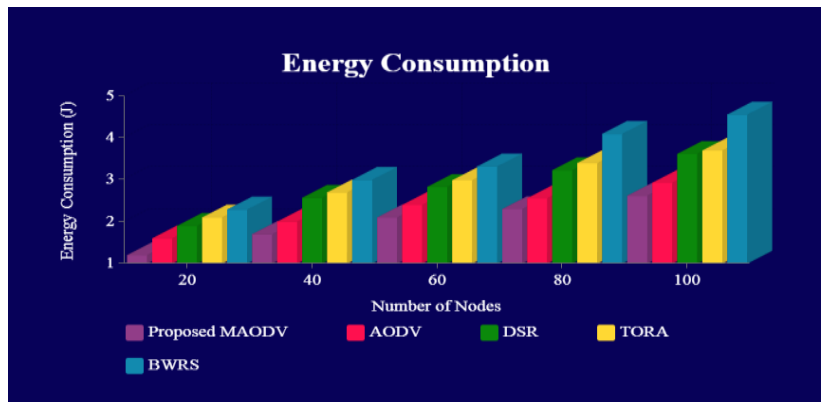

Fig. 7. Comparison of Energy Consumption for different Protocols.

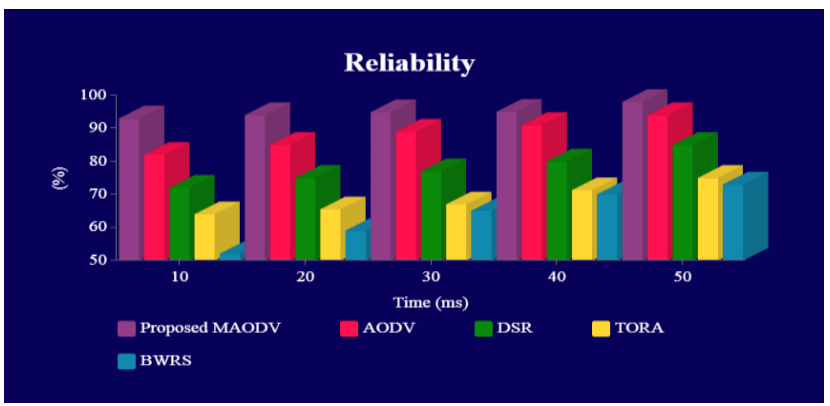

Fig. 8. Comparison of Reliability for different Protocols.

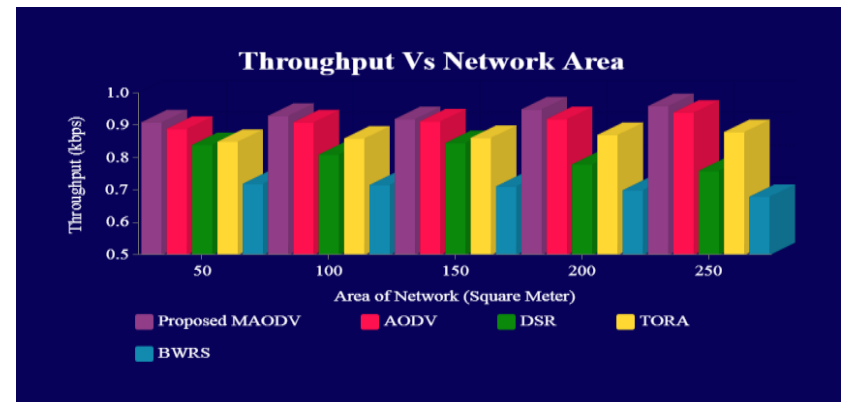

(a) Comparison of Throughput with Respect to Network Area for different Protocols.

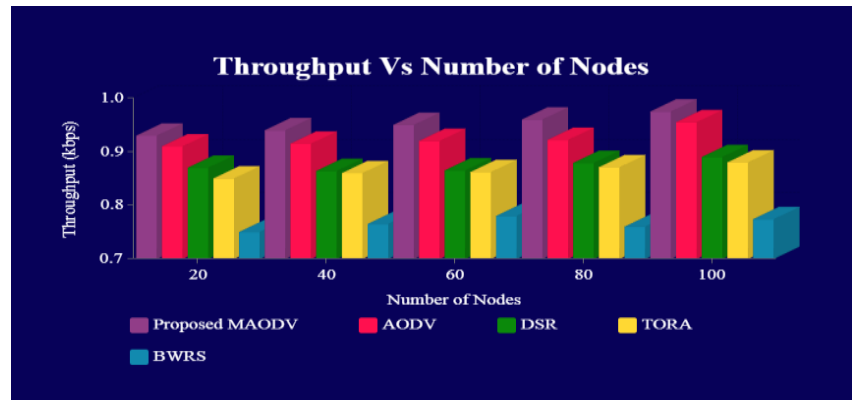

Fig. 9. (b) Comparison of Throughput with Respect to Number of Nodes for different Protocols.

Fig. 9(a) exhibits the TP analysis of the proposed with that of the prevailing protocols concerning network size. The MAODV attains a $0.93 \mathrm{kbps}$ TP when the number of nodes is 20. And for AODV, DSR, TORA protocol, the TP is $0.91 \mathrm{kbps}, \quad 0.87 \mathrm{kbps}, \quad 0.85 \mathrm{kbps}$, and $0.75 \mathrm{kbps}$, correspondingly, whereas for BWRS, the TP is $0.75 \mathrm{kbps}$. From which, it can be said that the TP of these prevailing 
protocols is lesser compared to the MAODV. The TP of MAODV is higher than others even for all the disparate nodes. And the Fig. 9(b) exhibits the TP of all the RP, which is gauged by means of differing network area. The proposed RP get a high TP compared to AODV, DSR, TORA, and BWRS protocol for disparate network area also. Thus, all the outcomes proved that the network is highly effective and consistent when utilizing MAODV.

\section{CONCLUSION}

The WANET's data communication performance has been rigorously affected by the $\mathrm{NC}$ that gives rise to network connection loss and data PL. The optimal routing centered load-balanced congestion control utilizing the MAODV method is projected in this work aimed at the WANET environment for evading problems like this. Here, the path comprising congested nodes has been picked out and then isolated. After that, the network traffic is dispersed over the congestion-free optimal routing path employing the MAODV for evading the packet drop and attaining effective DT. The proposed protocol's performance is examined and evaluated via the analogy of its performance with the existent method's performance. The analogy's result declared that the MAODV centered network attains higher reliability, higher TP, higher delivery ratio, less energy consumption, and less end to end delay. The network's energy consumption aimed at 100 nodes employing the MAODV has been just 2.62J. The MAODV's end to end delay is $0.019 \mathrm{~ms}$; it obtained a $96.9 \%$ higher packet delivery ratio. This performance analogy proves that the MAODV method is much proficient aimed at suppressing the NC in WANET. The cryptographic techniques might be involved in the proposed MAODV in the upcoming days aimed at evading attacks in DT.

\section{REFERENCES}

[1] Nandakumar, S. D, and Thirunadana Sikamani K, "Congestion and SINR evaluation for improving traffic capacity in Ad Hoc wireless networks", In IOP Conference Series: Materials Science and Engineering, vol. 925, no. 1, pp. 012070, 2020.

[2] Dimitris Kanellopoulos, "Congestion control for MANETs: An overview", ICT Express, vol. 5, no. 2, pp. 77-83, 2019.

[3] Rajesh, M., and Gnanasekar J. M, "Congestion control using aodv protocol scheme for wireless ad-hoc network", Advances in Computer Science and Engineering, vol. 16, no. 1/2, pp. 19, 2016.

[4] Chuang Ma, Jang-Ping Sheu, and Chao-Xiang Hsu, "A game theory based congestion control protocol for wireless personal area networks", Journal of Sensors, 2016, 10.1155/2016/6168535.

[5] Imtiaz Mahmud, Geon-Hwan Kim, Tabassum Lubna, and You-Ze Cho, "BBR-ACD: BBR with advanced congestion detection", Electronics, vol. 9, no. 1, pp. 136, 2020.

[6] Dionisis Kandris, George Tselikis, Eleftherios Anastasiadis, Emmanouil Panaousis, and Tasos Dagiuklas, "COALA: a protocol for the avoidance and alleviation of congestion in wireless sensor networks", Sensors, vol. 17, no. 11, pp. 2502, 2017.

[7] Tayyaba Abdul Haq, Khwaja Mansoor, and Saba Mahmood, "Congestion avoidance adaptive routing protocol for manets using network coding", In International Conference on Communication Technologies (ComTech), IEEE, pp. 47-52, 2019.

[8] Christian Lochert, Björn Scheuermann, and Martin Mauve, "A survey on congestion control for mobile ad hoc networks", Wireless communications and mobile computing, vol. 7, no. 5, pp. 655-676, 2007.

[9] Jiashuai Wang, Xiaoping Yang, Ying Liu, and Zhihong Qian, "A contention-based hop-by-hop bidirectional congestion control algorithm for Ad-Hoc networks", Sensors, vol. 19, no. 16, pp. 3484, 2019.
[10] Nousheen Akhtar, Muazzam A. Khan Khattak, Ata Ullah, and Muhammad Younus Javed, "Efficient routing strategy for congestion avoidance in MANETs", In 2017 International Conference on Frontiers of Information Technology (FIT), IEEE, pp. 305-309, 2017, 10.1109/FIT.2017.00061.

[11] Juan Pablo Astudillo León, Thomas Begin, Anthony Busson, and J. Luis, "A fair and distributed congestion control mechanism for smart grid neighborhood area networks", Ad Hoc Networks, vol. 104, pp. $102169,2020$.

[12] Rajesh Kumar, and Sudhir K. Routray, "Ant colony based dynamic source routing for VANET", In 2nd International Conference on Applied and Theoretical Computing and Communication Technology (iCATccT), IEEE, pp. 279-282, 2016.

[13] Bata Krishna Tripathy, Swagat Kumar Jena, Padmalochan Bera, and Satyabrata Das, "An adaptive secure and efficient routing protocol for mobile Ad Hoc networks", Wireless Personal Communications, 2020, 10.1007/s11277-020-07423-x.

[14] Rajesh, M., and Gnanasekar J. M, "Path observation based physical routing protocol for wireless ad hoc networks", Wireless Personal Communications, vol. 97, no. 1, pp. 1267-1289, 2017.

[15] Masaru Yoshimachi, and Yoshifumi Manabe, "A new AODV route discovery protocol to achieve fair routing for mobile ad hoc networks", In 6th International Conference on Information Communication and Management (ICICM), IEEE, pp. 222-226, 2016.

[16] Abdulaziz Al-Nahari, and Mohd Murtadha Mohamad, "Receiver-based ad hoc on demand multipath routing protocol for mobile ad hoc networks", Plos one, vol. 11, no. 6, pp. e0156670, 2016.

[17] Rakesh Kumar Sahu, and Narendra S. Chaudhari, "Energy reduction multipath routing protocol for MANET using recoil technique", Electronics, vol. 7, no. 5, pp. 56, 2018.

[18] Yefa Mai, Fernando Molina Rodriguez, and Nan Wang, "CC-ADOV: An effective multiple paths congestion control AODV", In IEEE 8th Annual Computing and Communication Workshop and Conference (CCWC), IEEE, pp. 1000-1004, 2018.

[19] Gagandeep Singh, Ashok Kumar Sharma, Onkar Singh Bawa, and Harneet Kaur, "Effective congestion control in MANET", In International Conference on Intelligent Engineering and Management (ICIEM), IEEE, pp. 86-90, 2020.

[20] Hui Wang, Junyong Tang, and Bo Hong, "Research of wireless congestion control algorithm based on EKF", Symmetry, vol. 12, no. 4, pp. 646, 2020.

[21] Devarajan Krishnamoorthy, Padmathilagam Vaiyapuri, Ayyasamy Ayyanar, Y. Harold Robinson, Raghvendra Kumar, Hoang Viet Long, and Le Hoang Son, "An effective congestion control scheme for MANET with relative traffic link matrix routing", Arabian Journal for Science and Engineering, pp. 1-11, 2020,10.1007/s13369-020-04511-9.

[22] Khalid Awan, M., Nadeem Ashraf, Muhammad Qaiser Saleem, Osama E. Sheta, Kashif Naseer Qureshi, Asim Zeb, Khalid Haseeb, and Ali Safaa Sadiq, "A priority-based congestion-avoidance routing protocol using IoT-based heterogeneous medical sensors for energy efficiency in healthcare wireless body area networks", International Journal of Distributed Sensor Networks, vol. 15, no. 6, 2019, pp. 1550147719853980.

[23] Vadivel, R., and V. Murali Bhaskaran, "Adaptive reliable and congestion control routing protocol for MANET", Wireless Networks, vol. 23, no. 3, pp. 819-829, 2017.

[24] Jogendra Kumar, Annapurna Singh, and H. S. Bhadauria, "Congestion control load balancing adaptive routing protocols for random waypoint model in mobile ad-hoc networks", Journal of Ambient Intelligence And Humanized Computing, 2020, 10.1007/s12652-020-02059-y.

[25] Gowtham, M. S., and Kamalraj Subramaniam, "Congestion control and packet recovery for cross layer approach in MANET", Cluster Computing, vol. 22, no. 5, pp. 12029-12036, 2019.

[26] Ammar Alhosainy, and Thomas Kunz, "Joint Optimal Congestion, Multipath Routing, and Contention Control for Wireless Ad Hoc Networks", IEEE Communications Letters, vol. 21, no. 12, pp. 26702673, 2017. 